[Letter]

\title{
Application of Logistic Function to Synthesis Process of MFI-type Silicalite from Quartz
}

\author{
Hideo Nagata $^{\dagger 1) *}$, Soichiro Kawahara ${ }^{\dagger 1)}$, Tetsuya Watanabe ${ }^{\dagger 1)}$, Yasuhiko Tanaka ${ }^{\dagger 1)}$, Hiroyasu Hamada ${ }^{\dagger 2)}$ \\ $\dagger 1)$ Dept. of Chemical and Biological Engineering, National Institute of Technology, Sasebo College, \\ Okishin-cho, Sasebo, Nagasaki 857-1193, JAPAN \\ $\dagger 2)$ Dept. of General Education, National Institute of Technology, Sasebo College, \\ Okishin-cho, Sasebo, Nagasaki 857-1193, JAPAN
}

(Received August 7, 2019)

\begin{abstract}
A logistic function was applied to analyze the synthesis of MFI-type silicalite from raw materials with different sodium hydroxide/quartz ratios. A linear equation $(f(t)=a t+b)$, a square-root equation $(f(t)=a \sqrt{ }(t)+$ $b)$, and a logarithmic equation $(f(t)=a \ln (t)+b)$ were used, all of which were functions of synthesis time. Parameters $a$ and $b$ were determined by the least-squares method. Using the logarithmic function $(f(t)=a \ln (t)+$ $b$ ) gave the highest correlation with the experimental results. The slope, $a$, was almost independent of the synthesis conditions, whereas the intercept, $b$, varied greatly. In addition, the values obtained for the length of the induction period and the crystal growth rate using the calculated parameters were in good agreement with the experimental results.
\end{abstract}

Keywords: MFI-type silicalite synthesis, Logistic regressive analysis, Logarithmic equation

\section{Introduction}

The synthesis time dependence of the degree of crystallinity of a substance such as a zeolite formed by dissolution and reprecipitation is well known to follow a sigmoidal curve. The degree of crystallinity can be determined by X-ray diffraction (XRD). Generally, the crystal formation process is divided into an induction period in which no clear peaks are present in the X-ray diffraction pattern, followed by a growth period in which

DOI: doi.org/10.1627/jpi.63.38

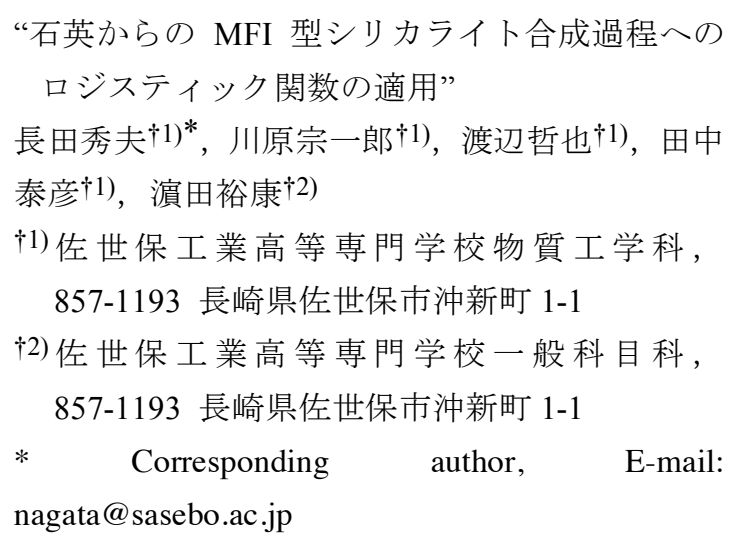

peaks begin to appear and their intensity increases with synthesis time. The induction period is the time from the start of the synthesis to the first appearance of peaks attributed to the zeolite product, and the subsequent rate of increase in peak intensity is an indication of the crystal growth rate.

The induction time and the subsequent growth rate are generally determined visually using time-dependence graphs, but the extent to which these values are used may differ greatly depending on the researcher. If the process could be carried out objectively, it would be possible to greatly reduce arbitrariness, and to easily compare results obtained by different researchers. This would also allow more accurate results to be obtained with smaller amounts of data.

In the field of mathematics, $S$-curves are represented by the following logistic function ${ }^{1), 2)}$ :

$$
y=\frac{1}{1+\exp (-f(x))}
$$

Here, $f(x)$ is generally a linear function of $x$. In addition, exp represents the power of Napier's constant. Figure 1 shows a plot of the logistic function $f(x)=x$. This 


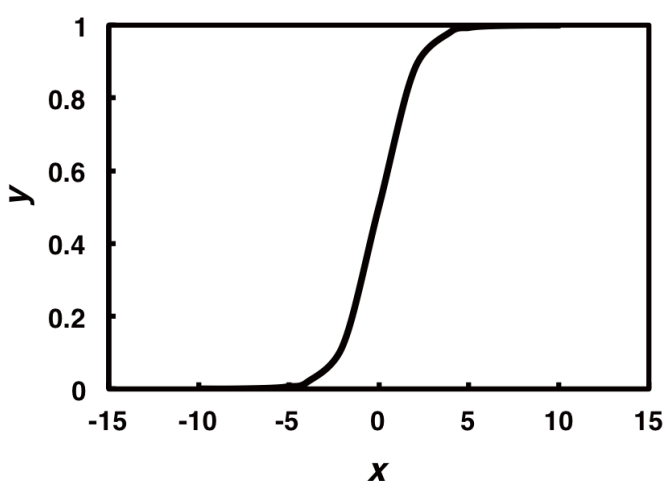

Fig. 1 Plot of Typical Logistic Function

function is seldom used in the field of chemistry but is often used in the fields of sociology 3 ),4), biology 5 ), and medicine/physiology 6 ). If the formation process for a crystalline substance can be described using this function, then the induction period and the crystal growth rate can be determined objectively.

Therefore, the present study investigated whether the synthesis of MFI-type silicalite from quartz ${ }^{7)}$ could be represented by a logistic function, and whether the induction period and growth rate could be determined.

\section{Experimental}

\section{1. Synthesis of MFI-type Silicalite}

MFI-type silicalite from quartz was synthesized using cylindrical stainless steel closed containers with an inner coating of Teflon, and volumes of $300 \mathrm{~mL}$ and 50 $\mathrm{mL}$. A mixture of quartz, sodium hydroxide, and water (total $300 \mathrm{~g})$ in a molar ratio of $1: x: 100(x=2,4$, 8) was sealed in the $300-\mathrm{mL}$ container, and quartz dissolution treatment was performed at $180{ }^{\circ} \mathrm{C}$ for $48 \mathrm{~h}$. Aliquots of the resulting solution were placed into six of the $50-\mathrm{mL}$ containers, the $\mathrm{pH}$ was adjusted to 10 by adding hydrochloric acid, and tetrapropylammonium bromide in an equimolar amount with quartz was dissolved in the solution. Hydrothermal synthesis was then carried out for 72 h. X-ray diffraction (XRD; Rigaku X-ray diffractometer) measurements were performed to identify the product and to calculate the degree of crystallinity based on the sum of the intensities of two peaks at $8-10^{\circ}$ and three peaks at $23-25^{\circ}$.

\section{2. Logistic Regression Analysis}

Regression analysis ${ }^{8)}$ was used to fit the logistic function to the crystallinity data obtained by XRD measurements during MFI-type silicalite synthesis. Assuming that the independent variable is the synthesis time $t[\mathrm{~h}]$ and the dependent variable is the degree of crystallinity $\mathrm{cr}$ [\%], Eq. (1) becomes

$$
\operatorname{cr}[\%]=\frac{100}{1+\exp (-f(x))}
$$

The inverse of this function is the logit function:

$$
\ln \left(\frac{c r}{100-c r}\right)=f(x)
$$

A regression analysis using a logit function is called logistic regression analysis. In the present study, the functions $f(t)=a t+b, f(t)=a \sqrt{ }(t)+b$, and $f(t)=a \ln (t)+$ $b$ were used. The parameters $a$ and $b$ were determined by the least-squares method using Microsoft Excel.

\section{Results and Discussion}

\section{1. Measurement of Formation rate of MFI-type Silicalite}

The MFI-type silicalite formation rate was measured using quartz heated and dissolved for $48 \mathrm{~h}$ in aqueous solutions with various sodium hydroxide concentrations. The degree of crystallinity of the MFI-type silicalite in the product was determined by comparison to the XRD peak intensity of a sample $(x=2)$ synthesized in $72 \mathrm{~h}$ to $100 \%$, which had high specific surface area (ca. 350 $\mathrm{m}^{2} / \mathrm{g}$ ) and sufficiently high crystallinity. The results are shown in Fig. 2. Higher sodium hydroxide concentration resulted in lower crystallinity of the MFI-type silicalite. That is, higher sodium hydroxide concentration in the raw material decreased the formation rate.

\section{2. Logistic Regression Analysis}

Logistic regression analysis was first performed on the results using the most common function, $f(t)=a t+b$. The correlation between the data and the regression equation was evaluated using the coefficient of determination, which is the square of the correlation coefficient and has a maximum value of 1 (indicating $100 \%$ correlation). Using $f(t)=a t+b$, the coefficient of 


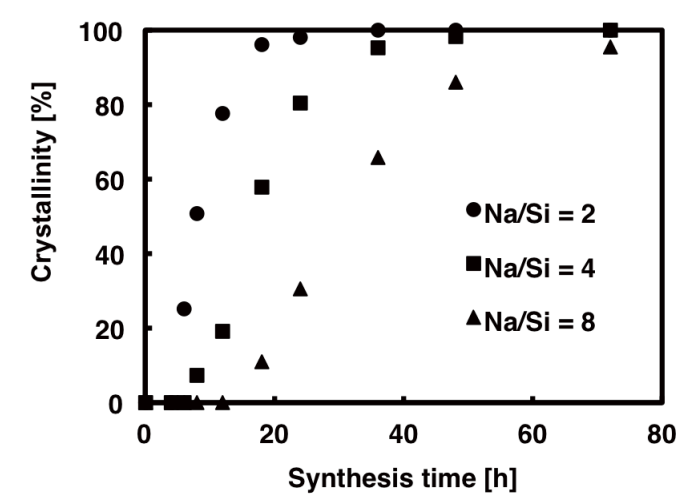

Fig. 2 Relationship between Crystallinity and Synthesis Time

determination ranged from 0.93 to 0.96 , indicating a fairly high correlation. Eq. (3) can be considered approximately linear for short synthesis times, but as the synthesis time increased, the data deviated downward from a straight line (Fig. 3). Therefore, logistic regression analysis was next performed using $f(t)=a \sqrt{ }(t)$ $+b$ and $f(t)=a \ln (t)+b$. Using $f(t)=a \sqrt{ }(t)+b$, the coefficient of determination was 0.97-0.99, which was an improvement over $f(t)=a t+b$. However, using $f(t)$ $=a \ln (t)+b$, the coefficient of determination was 0.991.00 indicating an extremely high correlation. Therefore, the obtained logit function was converted to a logistic function and compared with the experimental data.

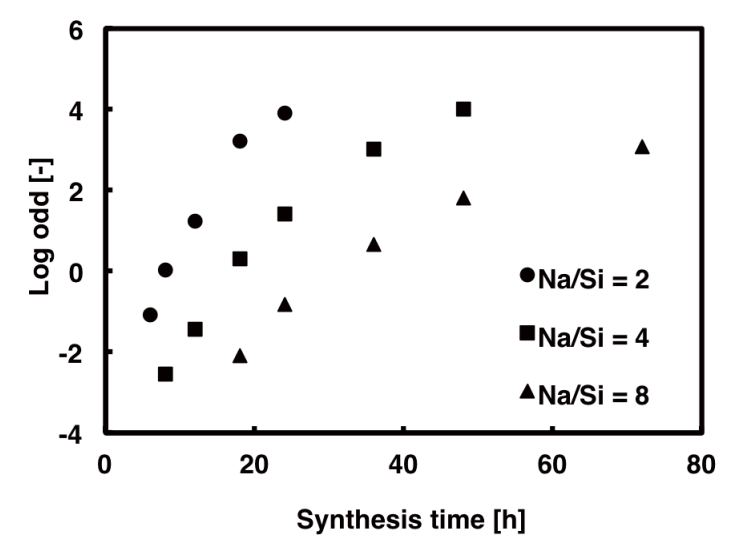

Fig. 3 Comparison between Natural Logarithm of Odd and Synthesis Time

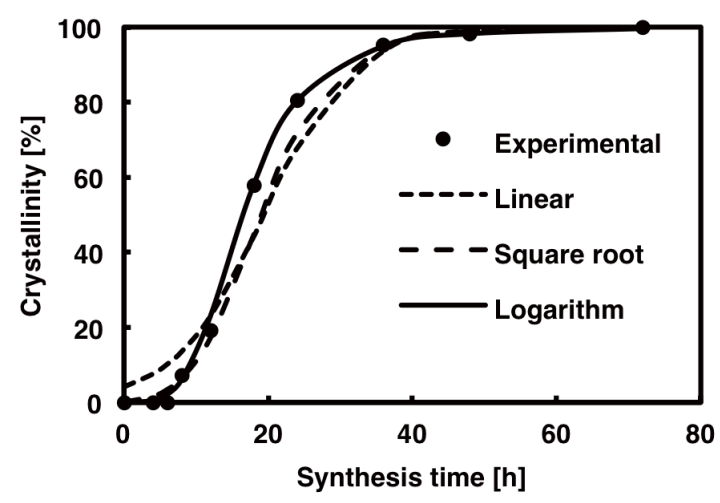

Fig. 4 Comparison between Experimental Data and Regression Analysis

The results for $x=4$ are shown in Fig. 4. Using $f(t)=a t+b$, an $S$-shaped curve was produced, but the degree of crystallinity predicted by the logistic function was not zero at $t=0$. The correlation between the experimental data and the logistic function was probably low because the crystal growth rate predicted by the logistic regression analysis was lower than expected from the experimental data. Using $f(t)=a \sqrt{ }(t)+b$, the predicted crystal growth rate from the experimental data was improved over the linear equation. However, the correlation between the experimental data and the logistic function was not very high. In contrast, using $f(t)=a \ln (t)+b$, the logistic function accurately reproduced the experimental data. The parameters obtained using the logarithmic function are summarized in Table 1. Parameter $a$ was almost independent of the sodium hydroxide/quartz molar ratio, whereas parameter $b$ decreased with higher sodium hydroxide/quartz molar ratio.

Table 1 Dependence of Logistic Function Parameters on Synthesis Conditions

\begin{tabular}{rrrr}
\hline & \multicolumn{3}{c}{ Na/Si ratio } \\
& \multicolumn{1}{c}{2} & \multicolumn{1}{c}{8} \\
\hline & & & \multicolumn{1}{c}{8} \\
\hline Parameter $a$ & 3.69 & 3.77 & 3.73 \\
Parameter $b$ & -7.70 & -10.56 & -12.74 \\
\hline
\end{tabular}




\section{3. Parameter Estimation}

The induction period and the crystal growth rate were estimated from the logistic function obtained using the logarithmic function with the highest correlation. First, the degree of crystallinity was assumed to have a linear dependence on the synthesis time $(c r=\alpha t+\beta)$ during the crystal growth stage. The slope of the assumed straight line was taken as a derivative when the degree of crystallinity is $50 \%$ and passed through a point with the degree of crystallinity of $50 \%$. The induction period and the crystal growth rate can be estimated from this linear equation. The induction period is the $x$-intercept and the crystal growth rate is the slope. With the time taken for the degree of crystallinity to reach $50 \%$ determined from the logit function, the equation becomes $t_{50}=\exp (-b / a)$. Differentiation of the logistic function $(c r=100 /[1+\exp \{-a \ln (t)-b\}])$ with respect to synthesis time gives $c r^{\prime}=0.01(a / t) c r(100-c r)$. Substituting $t_{50}$ into this derivative gives the slope of the line, which can be expressed as $\alpha=25 a \exp (b / a)$. Assuming that a straight line with this slope passes through the point at which the degree of crystallinity is $50 \%$, the $y$-intercept is $\beta=50-25 a$. If the induction period $\left(t_{\text {in }}\right)$ and the crystal growth rate $\left(r_{\text {ss }}\right)$ are determined from this line, then $t_{\text {in }}=(1-2 / a) \exp (-b / a)$ and $r_{\mathrm{cg}}=25 a \exp (b / a)$.

Figure 5 shows the results of substituting the obtained values for the regression coefficients $a$ and $b$ and comparing them with the actual measured values under each condition. Some variation was observed, but the induction period and the crystal growth rate were well reproduced for each condition. Although the regression coefficients followed a normal distribution, whether this is also the case for the induction period and crystal growth rate determined using these coefficients remains unclear. However, a more objective evaluation can be performed by this method because the obtained values can be subjected to nonparametric statistical analysis.

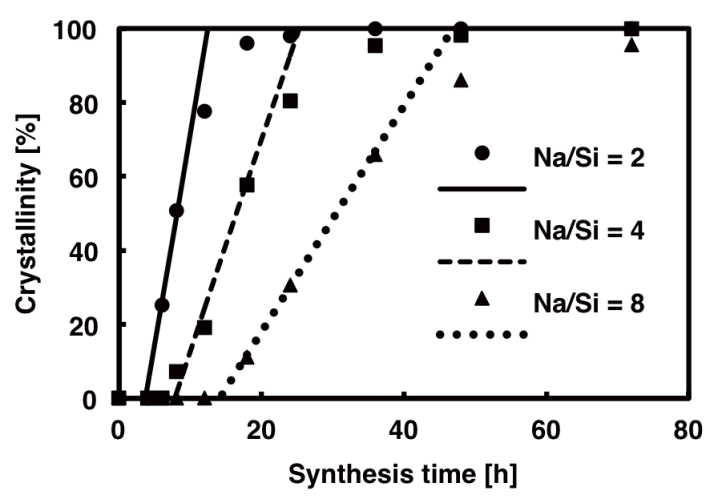

Fig. 5 Results of Substitution of Obtained Values of Regression Coefficients

\section{Conclusions}

Synthesis of MFI-type silicalite from quartz can be represented by a logistic function. The highest correlation was obtained using a logarithmic function of the synthesis time. The induction period and crystal growth rate could be predicted accurately using the obtained coefficients.

\section{References}

1) Verhulst, P.-F., Correspondance Mathématique et Physique, 10, 113 (1938).

2) Verhulst, P.-F., Nouveaux Mémoires de l'Académie Royale des Sciences et Belles-Lettres de Bruxelles, 18, 1 (1845).

3) Tsuzuki, K., Riron to Houhou, 17, 71 (2002).

4) Javanovic, B., Rob, R., Review of Economic Studies, 56, 569 (1989).

5) Shinozaki, S., Kagaku to Seibutsu, 17, 48 (1979).

6) Nakazawa, H., Nippon Ika Daigaku Igaku Kaishi, 10, 186 (2014).

7) Nagata, H., Kakimoto, S., Mori, H., J. Jpn. Petrol. Inst., 54, (6), 400 (2011).

8) Cox, D.R., J. Roy. Stat. Soc. B, 20, 215 (1958). 